\title{
MicroRNA-206 suppresses proliferation and predicts poor prognosis of HR-HPV-positive cervical cancer cells by targeting G6PD
}

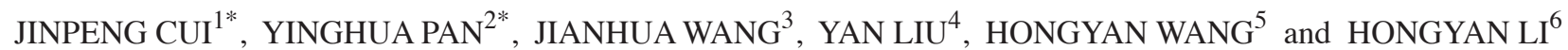 \\ ${ }^{1}$ Laboratory Medicine, Yantaishan Hospital; ${ }^{2}$ Imaging Department, Yantai Yuhuangding Hospital, Yantai, Shandong 264000; \\ ${ }^{3}$ Laboratory Medicine, People's Hospital of Rizhao, Rizhao, Shandong 276800; \\ Departments of ${ }^{4}$ Health Management and ${ }^{5}$ Obstetrics, People's Hospital of Zhangqiu District, Jinan, Shandong 250200; \\ ${ }^{6}$ Department of Outpatient, People's Hospital of Weifang, Weifang, Shandong 261000, P.R. China
}

Received January 4, 2018; Accepted August 2, 2018

DOI: $10.3892 / \mathrm{ol} .2018 .9326$

\begin{abstract}
Cervical cancer (CC) is one of the most frequent gynecological malignancies in females worldwide. Aberrant expression of microRNA (miR)-206 was reported to play an important role in tumor progression. The aim of the present study was to evaluate the potential role of miR-206 and verify its influence on the function of glucose-6-phosphate dehydrogenase (G6PD) in CC. Western blot analysis and RT-PCR were employed to measure miR-206 and G6PD expression. Luciferase assays were performed to validate G6PD as miR-206 targets. CCK-8 assay was performed to examine the regulation of miR-206 and G6PD on CC proliferation. The result showed that miR-206 was downregulated, while G6PD was upregulated in high risk human papillomavirus (HR-HPV)(+) CC. miR-206 directly targeted the 3'-UTR of G6PD. miR-206 overexpressed or G6PD low-expressed suppressed cell proliferation. miR-206 low expressed or G6PD overexpressed predicted poor prognosis. In conclusion, miR-206 reduced cancer growth and suppresses the G6PD expression in CC. This newly identified miR-206 may provide further insight into tumor progression and offers a promising target for the CC therapy.
\end{abstract}

\section{Introduction}

Cervical cancer (CC) remains one of the most frequent gynecological malignancies among females worldwide, which leads to the highest morbidity and mortality in young women,

Correspondence to: Dr Hongyan Li, Department of Outpatient, People's Hospital of Weifang, 5 Guangwen Street, Kuiwen, Weifang, Shandong 261000, P.R. China

E-mail: hf1669410@163.com

${ }^{*}$ Contributed equally

Key words: miR-206, G6PD, HR-HPV, cervical cancer particularly in developing country (1). Considered as the major etiologic contributor to the pathogenesis of CC, HPVs have been associated with more than $99 \%$ of cervical carcinomas (2). It is clear that persistent infection of high-risk human papillomavirus (HR-HPV), especially HPV18 and HPV16 are the most important etiologic agent in cervical carcinogenesis $(3,4)$. The viral oncoproteins HPV18 and HPV16 E7 and E6 can inactivate $\mathrm{pRB}$ and $\mathrm{p} 53$, thereby influencing their regulation and subsequently contributing to cell cycle checkpoint escape and cervical carcinogenesis $(5,6)$. However, due to the CC complex mechanism, the regulatory mechanism and the biological functions underlying HPV pathogenesis need to be further investigated.

Glucose-6-phosphate dehydrogenase (G6PD) catalyses the first rate-limiting step in the pentose phosphate pathway (PPP) (7). G6PD produces nicotinamide adenine dinucleotide phosphate (NADPH) that affect antioxidant defense and biosynthesis in the cells and is especially important in red blood cells functionally (8). G6PD is taken into account as one oncogene on account of its high expression in a great range of tumors, including breast cancers (9), melanoma (10), colorectal cancer (11) and lung cancer (12). A previous study has demonstrated that the expression of G6PD was high and there is a positive correlation with cervical patients infected with HPV18 and HPV16 of 30 to 40 years female (13). The high expression of G6PD may affect the progression and development of HR-HPV16/18-infected CC. Its underlying molecular mechanisms and the biological functions for its oncogenic roles in HR-HPV16/18 infected CC are still unknown.

MicroRNAs (miRNAs/miRs) are a class of highly conserved, non-coding and endogenous RNAs (ranging in 18-23 nucleotides length) $(14,15)$, which can modulate the physiological process or pathogenesis through partial complementary binding to the 3'-UTR of mRNAs (16). miR-206 has been demonstrated to be involved in different physiological and pathological processes (17). Dysfunctions of miR-206 occurred in a group of tumors, such as hepatocellular carcinoma (18), head and neck squamous cell carcinoma (19) and medulloblastoma (17), which can regulate tumor progression that is invovled in cell differentiation, proliferation, metastasis, 
and apoptosis. However, the specific functional molecular mechanisms of miR-206 in CC are still elusive, and the potential of miR-206 as a therapeutic target of $\mathrm{CC}$ remains to be evaluated.

We demonstrated that miR-206 was frequently downregulated while G6PD was upregulated in $\mathrm{HR}-\mathrm{HPV}(+) \mathrm{CC}$. Overexpression of miR-206 or low expressed G6PD suppressed cell proliferation, and miR-206 low expressed or G6PD overexpressed predicted poor prognosis. Furthermore, we identified G6PD as a direct target of miR-206. We also measured the overall survival (OS) according to the expression of miR-206 and G6PD. The newly identified miR-206/G6PD axis partially elucidates the molecular mechanism of proliferation and is a novel potential therapeutic target for CC treatment.

\section{Materials and methods}

Patient selection and human tissues. A total of $56 \mathrm{CC}$ patients (including 42 HPV16/18-positive CCs, 14 HPV-negative CCs) who were treated at the Yantaishan Hospital (Yantai, China) between March, 2014 and August, 2016 participated in this study. Written informed consent was provided by patients. The study was approved by the Ethics Committee of Yantaishan Hospital. All the tissues were independently and histologically diagnosed, and $\mathrm{CC}$ was classified based on the International Federation of Gynecology and Obstetrics (FIGO) staging system (20). All the specimens were stored at $-80^{\circ} \mathrm{C}$. PCR amplification was used to detect cervical HPV infection with the presence of HPV DNA (7).

Cell culture and treatments. HPV16-positive SiHa (HPV16 ${ }^{+} \mathrm{SiHa}$ ), and HPV18-positive HeLa (HPV18 ${ }^{+} \mathrm{HeLa}$ ) were purchased from the American Type Culture Collection (Manassas, VA, USA). The cells were cultured in DMEM (Gibco; Thermo Fisher Scientific, Inc., Waltham, MA, USA) supplemented with $10 \%$ FCS (Invitrogen; Thermo Fisher Scientific, Inc.) at $37^{\circ} \mathrm{C}$ and $5 \% \mathrm{CO}_{2}$.

miR-206 mimics, G6PD siRNA and the negative controls (NC) were obtained from GenePharma, Co., Ltd. (Shanghai, China). HeLa and $\mathrm{SiHa}$ cells were transfected with G6PD siRNA or miR-206 mimics as well as the NC using Lipofectamine 2000 Reagent (Invitrogen; Thermo Fisher Scientific, Inc.). Cells were used for proliferation after transfection. All transfection was conducted three times.

CCK-8 assay. CCK-8 (Dojindo Molecular Technologies, Inc., Kumamoto, Japan) was performed to detect cell proliferation. Cervical cells transfected with miR-206 mimics or G6PD siRNA were seeded into 96-well plates. Then, $10 \mu \mathrm{l} \mathrm{CCK-8}$ reagent was added to the wells. The absorbance of each well at 24, 48, 72 and $96 \mathrm{~h}$ was detected at $450 \mathrm{~nm}$.

Western blot analysis. Western blot analysis was used to detect the G6PD protein expression in HeLa and SiHa cells transfected with miR-206 mimics. Proteins were isolated from cervical cells with different transfections using RIPA lysis buffer (Thermo Fisher Scientific, Inc.). The protein was then transferred onto a PVDF membrane (Bio-Rad Laboratories, Inc., Hercules, CA, USA) and sealed with Tris-buffered saline Tween-20 (TBST). Then, the membranes were blocked by 5\% bovine serum albumin and incubated with specific primary antibody rabbit polyclonal anti-G6PD antibody (1:1,000; cat. no. ab993; Abcam, Cambridge, MA, USA) or GAPDH (1:3,000; cat. no. ab226408). After that, the membrane was incubated in the secondary antibody goat polyclonal anti-rabbit IgG H\&L secondary antibody (1:2,000; cat. no. ab150077; Abcam). The ECL detection system was used to detect the protein level (BestBio, Shanghai, China).

RNA isolation and RT-qPCR. Total RNA of cervical cells and tissues were extracted using TRIzol Reagent. RT-qPCR for miR-206/G6PD was performed with SYBR Premix Ex Taq ${ }^{\mathrm{TM}}$ (Takara Biotechnology Co., Ltd., Dalian, China). Relative gene expression was determined using the $2^{-\Delta \Delta \mathrm{Cq}}$ method (21). U6 and GAPDH acted as the internal control for the expressions of miR-206 and SPARC. G6PD and GAPDH primers were produced by Invitrogen; Thermo Fisher Scientific, Inc. The transcription primer and PCR primer of miR-206 and U6 were purchased from Guangzhou RiboBio Co., Ltd. (Guangzhou, China). Primer sequences were as follows: miR-206 forward, 5'-CCAAAGCGGAGTCTCGCAT-3' and reverse, 5'-GCCTAGCATCTTGCTTAGCTC-3'; U6 forward, 5'-GCTTCGGCAGCACATATACTAAAAT-3' and reverse, 5'-CGCTTCACGAATTTGCGTGTCAT-3'; G6PD forward, 5'-TGCCTTCCATCAGTCGGATACAC-3' and reverse, 5'-TGGTGGGGTAGATCTTCTTCTTGG-3'; and GAPDH forward, 5'-CCCTTCATTGACCTCAACTACATG-3' and reverse, 5'-TGGGATTTCCATTGATGACAAGC-3'.

Luciferase assay. The bioinformatics analysis software TargetScan (http://www.targetscan.org/) and miRanda (http://www.microrna.org/microrna/home.do) were chosen for predicting the targets of miR-206. For luciferase reporter, the wild-type (WT) and mutant type (Mut) 3'-UTR of G6PD were cloned into pcDNA3.1 vector (Ambion; Thermo Fisher Scientific, Inc.) and verified by sequencing. For the luciferase assay, the cells were co-transfected with miR-206 mimics and WT or Mut 3'-UTR of G6PD luciferase reporter plasmid. Then we used Dual-Luciferase Reporter Assay System (Promega Corporation, Madison, WI, USA) for measuring the reporter activities.

Statistical analysis. Statistical analyses were presented as the mean \pm standard deviation using SPSS19.0 software (SPSS, Inc., Chicago, IL, USA). Differences between groups were evaluated by Student's t-test or Tukey's post hoc test after ANOVA in SPSS. Correlation between mRNA and miRNA were estimated using the Spearman's correlation method. In addition, the Kaplan-Meier method with log-rank test was used for analyzing survival. $\mathrm{P}<0.05$ was considered to indicate a statistically significant difference.

\section{Results}

miR-206 is downregulated, while G6PD is upregulated in $H R-H P V(+) C C$. To investigate whether the miR-206 expression was altered in HR-HPV(+) cervical tissues, RT-qPCR was performed in $\mathrm{CC}$ and normal cervical tissues, obtained from 46 HPV16/18-positive patients and $10 \mathrm{HPV}$-negative patients. miR-206 expression was significantly lower in 
A
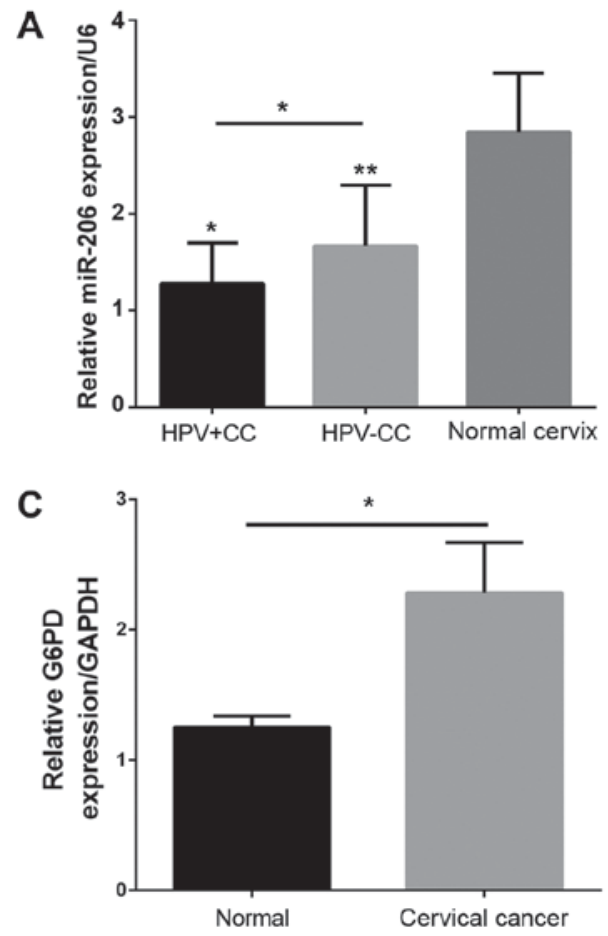

B

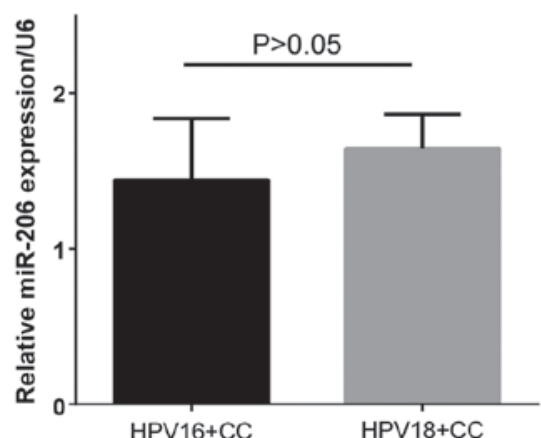

D

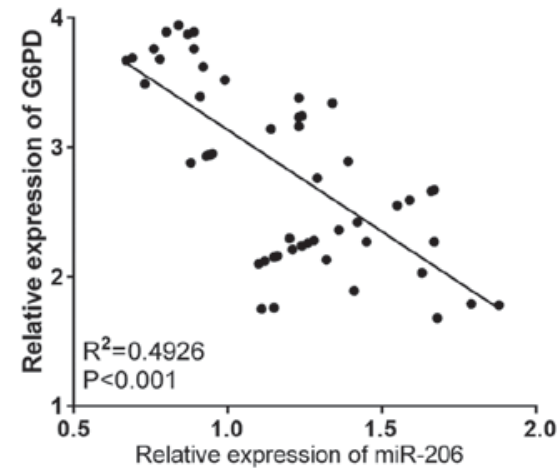

Figure 1. miR-206 was downregulated and inversely connected with G6PD. (A) miR-206 expression in CC tissues compared to normal cervical tissues detected by RT-qPCR. (B) miR-206 levels in HPV16(+) CC and HPV18(+) CC tissues. (C) Relative expression of G6PD in CC tissues. (D) Spearman's correlation analysis of miR-206 and G6PD expression in CC tissues. "P<0.05; ${ }^{* *} \mathrm{P}<0.01$. miR, microRNA; G6PD, glucose-6-phosphate dehydrogenase; HPV, human papillomavirus; $\mathrm{CC}$, cervical cancer.

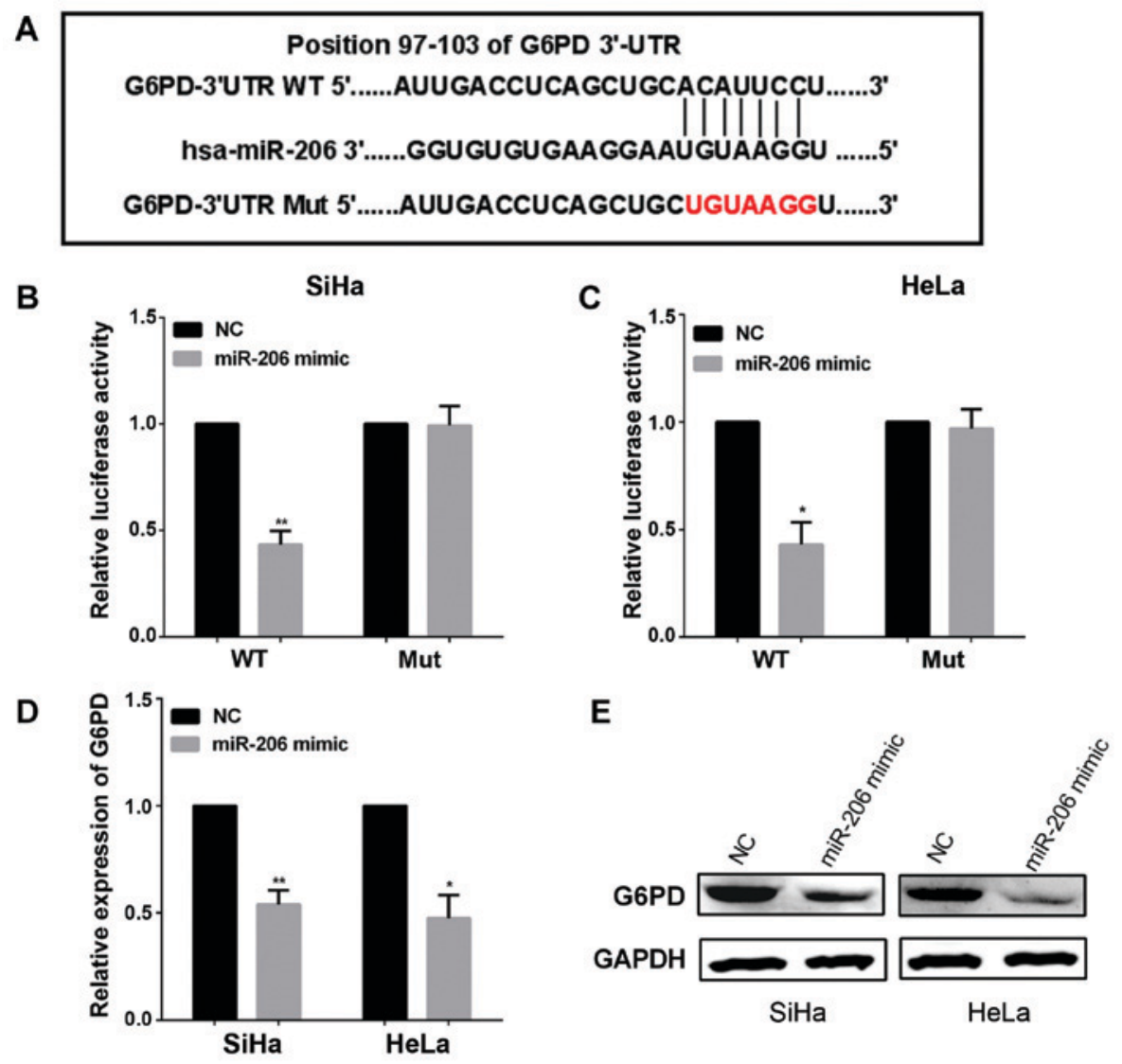

Figure 2. G6PD is the direct target of miR-206. (A) The binding sites of miR-206 on G6PD-3'-UTR. (B and C) Luciferase reporter assay with the pcDNA3.1-G6PD-3'-UTR-WT or pcDNA3.1-G6PD-3'-UTR-Mut were ransfected with miR-206 mimics. (D and E) G6PD protein level and mRNA level in HeLa and SiHa cells which transfected with miR-206 mimic. ${ }^{* * *} \mathrm{P}<0.01$, "P $<0.05$. G6PD, glucose-6-phosphate dehydrogenase; miR, microRNA; NC, negative controls; Mut, mutant type; WT, wild-type. 

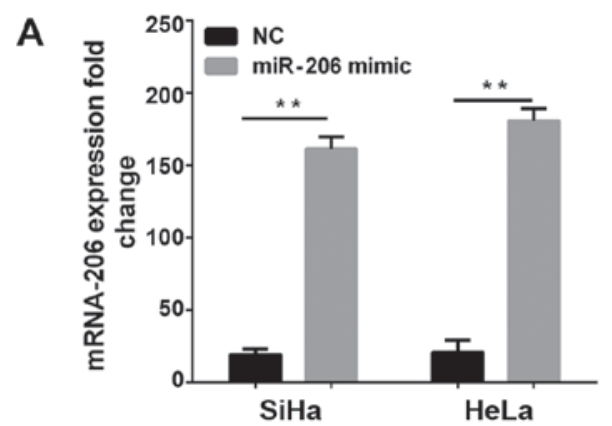

B

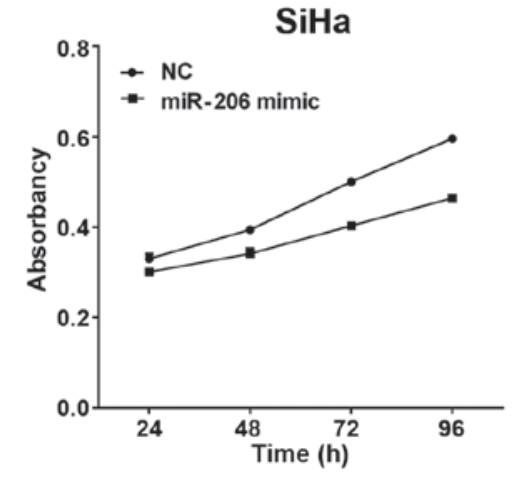

C

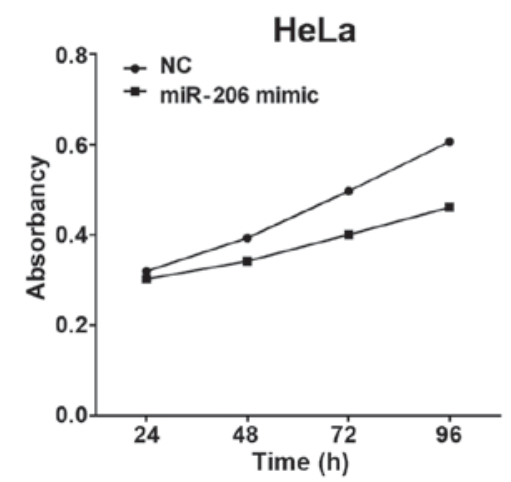

D

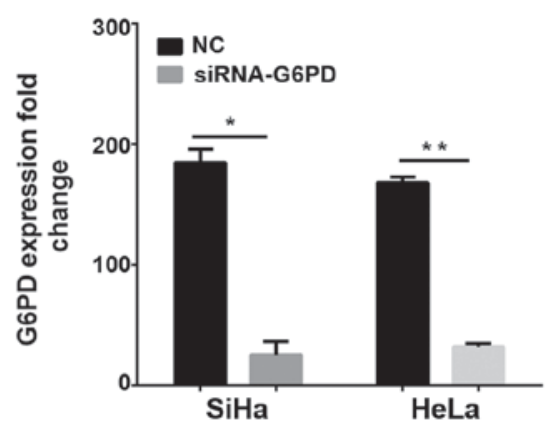

E

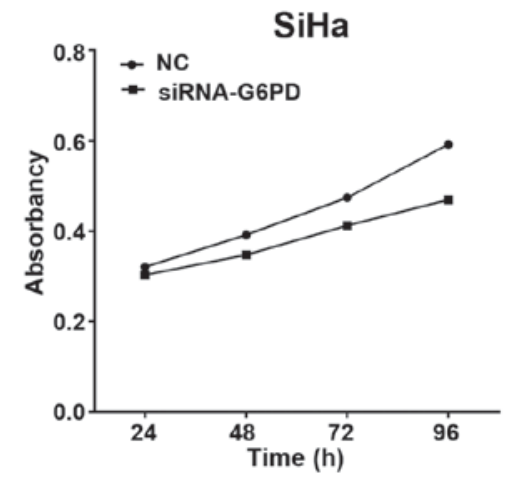

$\mathbf{F}$

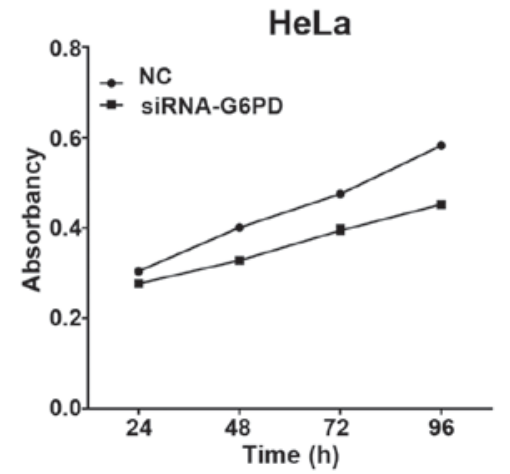

Figure 3. miR-206 overexpressed or G6PD low expressed suppressed cell proliferation. (A) miR-206 was overexpressed in CC cells. (B and C) miR-206 high expression suppressed CC cell proliferation. (D) Interfered G6PD expression by siRNA. (E and F) G6PD low expression suppressed CC cell proliferation. ${ }^{* *} \mathrm{P}<0.01,{ }^{*} \mathrm{P}<0.05$. miR, microRNA; G6PD, glucose-6-phosphate dehydrogenase; CC, cervical cancer; NC, negative controls.

CC tissues (Fig. 1A), and miR-206 expression was lower in HPV16/18-positive CC compared to HPV-negative tissues (Fig. 1A). However, the results showed no significant difference between HPV18(+) CC tissues $(n=20)$ and HPV16(+) CC tissues (n=26) (Fig. 1B). Generally, G6PD level was significantly higher in CC patients compared to normal control patients (Fig. 1C). An inverse correlation between miR-206 expression and the G6PD level in these clinical specimens $\left(\mathrm{R}^{2}=0.4926, \mathrm{P}<0.0001\right)$ (Fig. 1D). Thus, miR-206 and G6PD may have possible roles in modulating the progression of HR-HPV(+) CC.

miR-206 directly targets the 3'-UTR of G6PD. We predicted that G6PD was a downstream target of miR-206 by bioinformatics analysis software TargetScan and miRanda, the binding site of G6PD was at its 3'-UTR located at 97 to 103 as shown in Fig. 2A. To further confirm whether the 3'-UTR of G6PD can be directly targeted by miR-206, we performed luciferase reporter assay. Following the protocol, G6PD 3'-UTR-WT and G6PD 3'-UTR-Mut were cloned into plasmids with either
miR-206 mimic or NC, then following by the measurement of luciferase reporter assays. Luciferase activity decreased when miR-206 mimics were co-transfected with the G6PD 3'-UTR-WT plasmid $(\mathrm{P}<0.01)$, but there was no change with the G6PD 3'-UTR-Mut plasmid ( $\mathrm{P}>0.05)$ in $\mathrm{SiHa}$ and HeLa cells (Fig. 2B and C). These results suggested miR-206 can downregulate the expression of G6PD by binding to its predicted regions of 3'-UTR. Furthermore, when overexpressed miR-206 by transfected miR-206 mimic, the expression of G6PD was decreased in $\mathrm{SiHa}$ and HeLa cell (Fig. 2D). In Fig. 2E, overexpression of miR-206 reduced the protein level of G6PD in the $\mathrm{SiHa}$ and HeLa cells. Together, these results demonstrated miR-206 negatively regulated endogenous G6PD expression.

miR-206 overexpressed or G6PD low expressed suppressed cell proliferation. Due to the downregulation of miR-206 and its inverse correlation with G6PD, we hypothesized that miR-206 is a tumor suppressor of CC, and affected CC cell proliferation. For the sake of testing the impact of miR-206 on proliferation, we utilized miR-206 mimic to overexpress 
Table I. HPV status and miR-206 levels in women diagnosed as cervical cancer $(\mathrm{n}=56)$.

\begin{tabular}{|c|c|c|c|c|}
\hline \multirow[b]{2}{*}{ Variable } & \multicolumn{2}{|c|}{ HPV status (HR-HPV16/18) } & \multicolumn{2}{|c|}{ miR-206 expression } \\
\hline & $\begin{array}{c}\text { HPV 16 /18 } \\
\text { negative, n }(\%)\end{array}$ & $\begin{array}{c}\text { HPV } 16^{+} / 18^{+} \\
\text {positive, } \mathrm{n}(\%)\end{array}$ & $\begin{array}{l}\text { miR-206 low level } \\
(\leq \text { median), } \mathrm{n}(\%)\end{array}$ & $\begin{array}{l}\text { miR-206 high level } \\
(>\text { median }), \mathrm{n}(\%)\end{array}$ \\
\hline \multicolumn{5}{|l|}{ FIGO stage } \\
\hline I & $2(3.6)$ & $2(3.6)$ & $24(42.8)$ & $2(3.6)$ \\
\hline II & $4(7.1)$ & $8(14.3)$ & $14(25)$ & $2(3.6)$ \\
\hline III & $2(3.6)$ & $12(21.4)$ & $6(10.7)$ & $2(3.6)$ \\
\hline IV & $6(10.7)$ & $20(35.7)$ & $2(3.6)$ & $4(7.1)$ \\
\hline \multicolumn{5}{|c|}{ Differentiation } \\
\hline Well & $4(7.1)$ & $8(14.3)$ & $30(53.6)$ & $2(3.6)$ \\
\hline Moderate & $2(3.6)$ & $20(35.7)$ & $13(23.2)$ & $2(3.6)$ \\
\hline Poor & $8(14.3)$ & $12(21.4)$ & $3(5.4)$ & $6(10.7)$ \\
\hline \multicolumn{5}{|c|}{ Tumor diameter $(\mathrm{cm})$} \\
\hline$\leq 4$ & $10(17.8)$ & $19(33.9)$ & $32(57.1)$ & $8(14.3)$ \\
\hline$>4$ & $4(7.1)$ & $23(41.2)$ & $14(25)$ & $2(3.6)$ \\
\hline
\end{tabular}

miR, microRNA; HR-HPV, high risk human papillomavirus; FIGO, International Federation of Gynecology and Obstetrics.

A

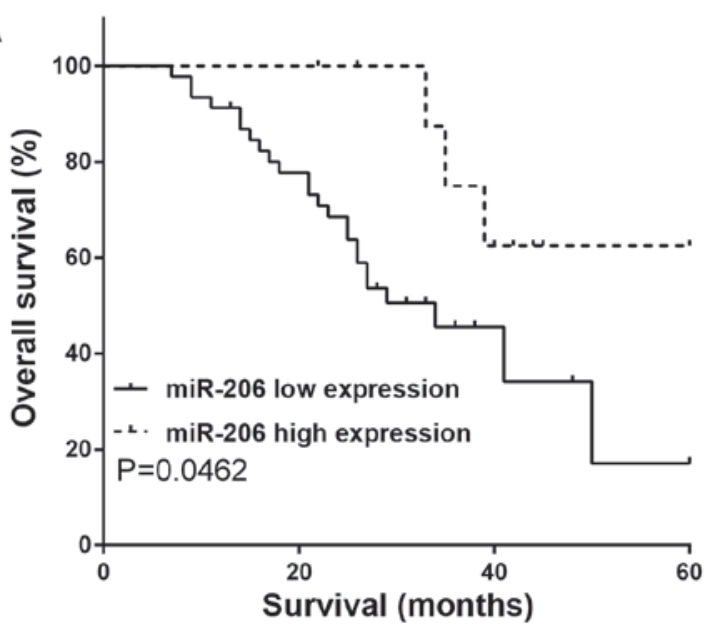

B

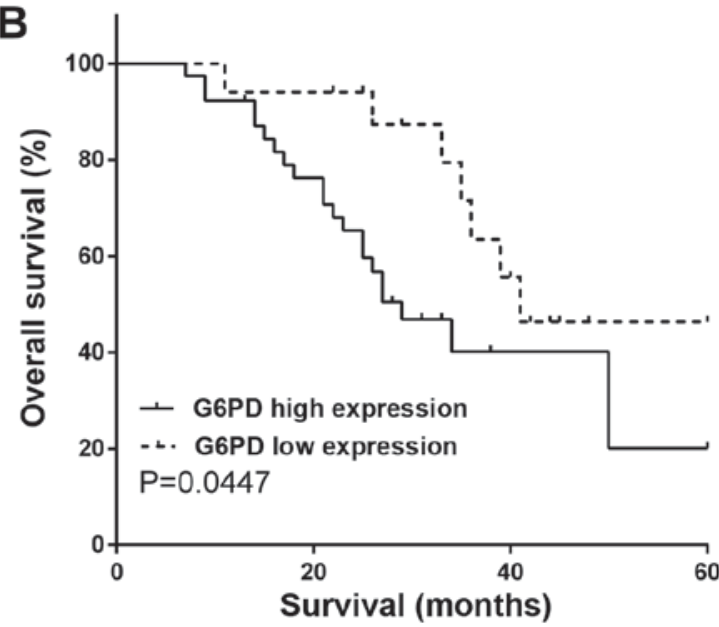

Figure 4. miR-206 low expressed or G6PD overexpressed predicted poor prognosis. (A) OS was calculated between patients according to the expression of miR-206. (B) OS was calculated on the basis of the expression of G6PD. miR, microRNA; G6PD, glucose-6-phosphate dehydrogenase; OS, overall survival.

miR-206 in CC SiHa and HeLa cells and then the expression levels in cells were determined by RT-qPCR (Fig. 3A). Then, we measured cell proliferative ability and found that for overexpressed miR-206 the proliferation ability was decreased both in SiHa and HeLa (Fig. 3B and C). To examine the effect of G6PD on the proliferation of CC, we used siRNA-G6PD to interfere with G6PD expression and the results $(\mathrm{P}<0.01)$ were measured by RT-qPCR, as shown in Fig. 3D, and then we calculated the capabilities of cell proliferation. Under these conditions, the results indicated cell proliferative ability was inhibited (Fig. 3E and F).

miR-206 low expressed or G6PD overexpressed predicted poor prognosis. We divided 56 gastric cancer patients into the miR-206 high expression group $(n=10)$ and miR-206 low expression group ( $\mathrm{n}=46)$ according to miR-206 expression level. In addition, the 56 patients were separated into HPV16 $/ 18^{-}$negative group $(n=14)$ and $\mathrm{HPV}^{+} 6^{+} / 18^{+}(\mathrm{n}=42)$ according to HPV status. The 56 patients were separated on the basis of FIGO stage, differentiation, tumor diameter, respectively, and the detailed grouping is shown in Table I.

To further evaluate whether miR-206 levels were associated with CC prognosis, we performed Kaplan-Meier analysis to evaluate five year OS in CC. OS was significantly poorer in patients with low tissue miR-206 expression than those with high miR-206 expression (log-rank, $\mathrm{P}=0.0462$; Fig. 4A). In addition, we measured OS according to G6PD expression, and the opposite results were obtained, whereby the OS was lower with G6PD overexpression compared with low expression (log-rank, $P=0.0447$; Fig. 4B). 


\section{Discussion}

$\mathrm{CC}$ was once considered to be one of the most serious cancers in women worldwide, and almost $90 \%$ of CC deaths occurred in developing countries of the world (22). Although cancer treatments have been improved in recent years, the outcomes of patients with CC remain unsatisfactory (23). Thus, identifying new targets for the development of effective therapeutics for CC is urgent. Dysregulation of miRNAs may lead to uncontrolled and progressive cancer growth and has been thoroughly reported in almost all types of human malignancies $(24,25)$, including CC (26). In this study, we found miR-206 was significantly downregulated in CC tissues, and was reduced in $\mathrm{HPV} 16^{+} / 18^{+} \mathrm{CC}$. However, there was no significant difference between HPV16(+) CC tissues and HPV18(+) CC tissues. Moreover, G6PD was identified as a direct target of miR-206 and the inverse relationship between them was also observed. We demonstrated that miR-206/G6PD may act as a novel potential therapeutic target and treatment for CC, and a low expression of miR-206 may contribute to tumor progression and cell proliferation in CC patients.

Accumulating evidence has shown that miRNAs can function as a crucial point in gene expressions, and then influence tumor development and progression (27). Mounting evidences have demonstrated that miR-206 is downregulated in breast cancer (28), gastric cancer (29) and various types of human tumors. The low expression of miR-206 may be linked with physiological and pathological processes of tumors, as those researchers proposed. A previous study has reported that miR-206 was downregulated and inhibited cell proliferation, invasion and migration in CC (30), but the underlying molecular mechanisms are still elusive. Our findings were consistent with all the findings, as we have demonstrated that the overexpression of miR-206 could inhibit proliferation by directly targeting G6PD in SiHa and HeLa. In addition, we identified that miR-206 downregulation and/or G6PD upregulation predicted poor prognosis.

This study revealed the relative expression of G6PD was higher in HPV16 ${ }^{+} / 18^{+} \mathrm{CC}$ tissues. G6PD can be found widely expressed in tumors and could function as an important member in regulating cell invasiveness, survival and oxidative stress $(10,31)$. The bioinformatics analysis software was used for predicting the targets of miR-206. And then G6PD was looked for as the potential gene effectors which may participate in the function of miR-206. We confirmed that G6PD was a direct target of miR-206, and it was confirmed that a higher G6PD expression played a significant role in tumor proliferation and predicted poor prognosis. G6PD may therefore be independent prognostic factors for OS of patients suffering from CC.

In conclusion, we have indicated that miR-206 acts as a tumor suppressor in $\mathrm{CC}$ by inhibiting cancer proliferation. Furthermore, we demonstrated that miR-206 has an inverse correlation with G6PD and directly targets it. This newly identified miR-206 may provide new insight into the progression of $\mathrm{CC}$ and offer a promising therapeutic target for the treatment of CC. Nevertheless, further investigation to examine the function miR-206/G6PD axis in tumorigenesis and progression of $\mathrm{CC}$ is needed.

\section{Acknowledgements}

Not applicable.

\section{Funding}

No funding was received.

\section{Availability of data and materials}

The datasets used and/or analyzed during the present study are available from the corresponding author on reasonable request.

\section{Authors' contributions}

JC and YP contributed to the study design, data acquisition and analysis and drafted the manuscript; JW and YL contributed to the conception of the study. HW and HL contributed significantly to the data analysis and study preparation. All authors have read and approved the final study.

\section{Ethics approval and consent to participate}

Written informed consent was obtained from all the patients. The study was approved by the Ethics Committee of Yantaishan Hospital (Yantai, China).

\section{Patient consent for publication}

Not applicable.

\section{Competing interests}

The authors declare that they have no competing interests.

\section{References}

1. Siegel RL, Miller KD and Jemal A: Cancer statistics, 2016. CA Cancer J Clin 66: 7-30, 2016.

2. Munagala R, Kausar H, Munjal C and Gupta RC: Withaferin A induces p53-dependent apoptosis by repression of HPV oncogenes and upregulation of tumor suppressor proteins in human cervical cancer cells. Carcinogenesis 32: 1697-1705, 2011.

3. zur Hausen H: Papillomaviruses and cancer: From basic studies to clinical application. Nat Rev Cancer 2: 342-350, 2002.

4. Moody CA and Laimins LA: Human papillomavirus oncoproteins: Pathways to transformation. Nat Rev Cancer 10: 550-560, 2010.

5. Woodman CB, Collins SI and Young LS: The natural history of cervical HPV infection: Unresolved issues. Nat Rev Cancer 7: $11-22,2007$.

6. Wang X, Wang HK, McCoy JP, Banerjee NS, Rader JS, Broker TR, Meyers C, Chow LT and Zheng ZM: Oncogenic HPV infection interrupts the expression of tumor-suppressive miR-34a through viral oncoprotein E6. RNA 15: 637-647, 2009.

7. Hu T, Li YS, Chen B, Chang YF, Liu GC, Hong Y, Chen HL and Xiyang YB: Elevated glucose-6-phosphate dehydrogenase expression in the cervical cancer cases is associated with the cancerigenic event of high-risk human papillomaviruses. Exp Biol Med (Maywood) 240: 1287-1297, 2015.

8. Salimi A, Paeezi M, Yousefsani BS, Shadnia S, HassanianMoghaddam $\mathrm{H}$, Zamani $\mathrm{N}$ and Pourahmad J: Inhibition of glucose-6-phosphate dehydrogenase protects hepatocytes from aluminum phosphide-induced toxicity. Pestic Biochem Physiol 143: 141-146, 2017.

9. Polat MF, Taysi S, Gul M, Cikman O, Yilmaz I, Bakan E and Erdogan F: Oxidant/antioxidant status in blood of patients with malignant breast tumour and benign breast disease. Cell Biochem Funct 20: 327-331, 2002. 
10. Hu T, Zhang C, Tang Q, Su Y, Li B, Chen L, Zhang Z, Cai T and Zhu Y: Variant G6PD levels promote tumor cell proliferation or apoptosis via the STAT3/5 pathway in the human melanoma xenograft mouse model. BMC Cancer 13: 251, 2013.

11. Pes GM, Bassotti G and Dore MP: Colorectal cancer mortality in relation to glucose - 6 - phosphate dehydrogenase deficiency and consanguinity in Sardinia: A spatial correlation analysis. Asian Pac J Cancer Prev 18: 2403-2407, 2017.

12. Giatromanolaki A, Sivridis E, Arelaki S and Koukourakis MI Expression of enzymes related to glucose metabolism in non-small cell lung cancer and prognosis. Exp Lung Res 43: 167-174, 2017.

13. Hu T, Chang YF, Xiao Z, Mao R, Tong J, Chen B, Liu GC, Hong Y, Chen HL, Kong SY, et al: miR-1 inhibits progression of high-risk papillomavirus-associated human cervical cancer by targeting G6PD. Oncotarget 7: 86103-86116, 2016.

14. Ambros V and Lee RC: Identification of microRNAs and other tiny noncoding RNAs by cDNA cloning. Methods Mol Biol 265: $131-158,2004$

15. Gimpel C, Avni FE, Bergmann C, Cetiner M, Habbig S Haffner D, König J, Konrad M, Liebau MC, Pape L, et al: Perinatal diagnosis, management, and follow-up of cystic renal diseases: A clinical practice recommendation with systematic literature reviews. JAMA Pediatr 172: 74-86, 2018.

16. Calin GA and Croce CM: MicroRNA signatures in human cancers. Nat Rev Cancer 6: 857-866, 2006.

17. Pan X, Wang Z, Wan B and Zheng Z: MicroRNA-206 inhibits the viability and migration of medulloblastoma cells by targeting LIM and SH3 protein 1. Exp Ther Med 14: 3894-3900, 2017.

18. Pang C, Huang G, Luo K, Dong Y, He F, Du G, Xiao M and Cai W: miR-206 inhibits the growth of hepatocellular carcinoma cells via targeting CDK9. Cancer Med 6: 2398-2409, 2017.

19. Liu F, Zhao X, Qian Y, Zhang J, Zhang Y and Yin R: MiR-206 inhibits head and neck squamous cell carcinoma cell progression by targeting HDAC6 via PTEN/AKT/mTOR pathway. Biomed Pharmacother 96: 229-237, 2017.

20. FIGO Committee on Gynecologic Oncology: FIGO staging for carcinoma of the vulva, cervix, and corpus uteri. Int J Gynaecol Obstet 125: 97-98, 2014

21. Livak KJ and Schmittgen TD: Analysis of relative gene expression data using real-time quantitative PCR and the 2(-Delta Delta C(T)) Method. Methods 25: 402-408, 2001.
22. Torre LA, Bray F, Siegel RL, Ferlay J, Lortet-Tieulent J and Jemal A: Global cancer statistics, 2012. CA Cancer J Clin 65 $87-108,2015$

23. Rob L, Halaska M and Robova H: Nerve-sparing and individually tailored surgery for cervical cancer. Lancet Oncol 11 292-301, 2010.

24. Pandima Devi K, Rajavel T, Daglia M, Nabavi SF, Bishayee A and Nabavi SM: Targeting miRNAs by polyphenols: Novel therapeutic strategy for cancer. Semin Cancer Biol 46: 146-157, 2017.

25. Muluhngwi $\mathrm{P}$ and Klinge CM: Identification of miRNAs as biomarkers for acquired endocrine resistance in breast cancer. Mol Cell Endocrinol 456: 76-86, 2017.

26. Chuanyin L, Xiaona W, Zhiling Y, Yu Z, Shuyuan L, Jie Y, Chao H, Li S, Hongying Y and Yufeng Y: The association between polymorphisms in microRNA genes and cervical cancer in a Chinese Han population. Oncotarget 8: 87914-87927, 2017.

27. Manikandan J, Aarthi JJ, Kumar SD and Pushparaj PN: Oncomirs: The potential role of non-coding microRNAs in understanding cancer. Bioinformation 2: 330-334, 2008.

28. Yin K, Yin W, Wang Y, Zhou L, Liu Y, Yang G, Wang J and Lu J: MiR-206 suppresses epithelial mesenchymal transition by targeting TGF- $\beta$ signaling in estrogen receptor positive breast cancer cells. Oncotarget 7: 24537-24548, 2016.

29. Yan B, Zhu CD, Guo JT, Zhao LH and Zhao JL: miR-206 regulates the growth of the teleost tilapia (Oreochromis niloticus) through the modulation of IGF-1 gene expression. J Exp Biol 216: 1265-1269, 2013

30. Ling S, Ruiqin M, Guohong Z, Bing S and Yanshan C: Decreased microRNA-206 and its function in cervical cancer. Eur J Gynaecol Oncol 36: 716-721, 2015.

31. Gao LP, Cheng ML, Chou HJ, Yang YH, Ho HY and Chiu DT: Ineffective GSH regeneration enhances G6PD-knockdown Hep G2 cell sensitivity to diamide-induced oxidative damage. Free Radic Biol Med 47: 529-535, 2009.

This work is licensed under a Creative Commons Attribution-NonCommercial-NoDerivatives 4.0 International (CC BY-NC-ND 4.0) License. 